\title{
Qi-Deficiency, Qi-Stagnation, Qi-Flowback, Blood Deficiency, and Fluid Retention in Patients with Depression, Psychiatric Disorders, or Psychosomatic Disorders
}

\author{
Tetsuya Kondo ${ }^{1}$, Shoji Tokunaga ${ }^{2}$, Hideyo Sugahara ${ }^{3}$, Kouichi Yoshimasu ${ }^{4}$, \\ Mariko Akamine ${ }^{5}$, Yoshio Kanemitsu ${ }^{6}$, Kanichiro Fujisawa ${ }^{7}$, \\ Yukiko Masuda $^{8}$, Chiharu Kubo ${ }^{6}$ \\ ${ }^{1}$ Kansai University of Health Sciences, Kumatori, Osaka, Japan; \\ ${ }^{2}$ Department of Preventive Medicine, Graduate School of Medical Sciences, \\ Kyushu University, Fukuoka, Japan; \\ ${ }^{3}$ Sugahara Tenjin Clinic, Fukuoka, Japan; \\ ${ }^{4}$ Department of Hygiene, School of Medicine, Wakayama Medical University, \\ Wakayama, Japan \\ ${ }^{5}$ Yamamoto Hospital, Beppu, Japan; \\ ${ }^{6}$ Department of Psychosomatic Medicine, Graduate School of Medical Sciences, \\ Kyushu University, Fukuoka, Japan; \\ ${ }^{7}$ Fukuoka Prison Clinic, Fukuoka, Japan; \\ ${ }^{8}$ Masuda Orthopedic and Medical Clinic, Fukuoka, Japan. \\ Email: kondo0724538409@kansai.ac.jp \\ Received May $13^{\text {th }}, 2011$; revised July $8^{\text {th }}, 2011$; accepted August $21^{\text {rd }}, 2011$.
}

\begin{abstract}
The purpose of this study is to clarify the Qi-blood-fluid patterns of psychiatric disorders such as depression, anxiety disorders, eating disorders, somatoform disorders and psychosomatic disorders such as irritable bowel syndrome, functional dyspepsia, etc. according to oriental medicine. The subjects were all new cases who consulted the psychosomatic clinic of Kyushu University Hospital from June 2000 to March 2001. Qi-deficiency, Qi-stagnation, Qi-flowback, blood deficiency, and fluid retention scores were calculated from the chief complaints and answers to the 102 questions of the Kyudai Medical Index (KMI), according to Terasawa's Qi-blood-fluid scoring system. Since the results of physical examinations were not investigated, items requiring physical examination in Terasawa's criteria were excluded from the calculation. The Qi-flowback, blood deficiency and fluid retention scores were significantly higher in females than males. Strong correlations were observed between Qi-deficiency and Qi-stagnation in males and between blood deficiency and fluid retention in both sexes. Multiple logistic regression analysis revealed that Qi-deficiency and Qi-stagnation in both sexes, especially Qi-deficiency, in males, were associated with major depression. Qi-flowback in females only and blood deficiency in males only were associated with anxiety disorders or a high State-trait Anxiety Inventory score. In females, Qi-flowback was also associated with somatoform disorders and irritable bowel syndrome. These results may be of assistance in treating occidental diseases in an oriental manner.
\end{abstract}

Keywords: Generalized Anxiety Disorder, Irritable Bowel Syndrome, Major Depression, Self-Rating Depression Scale (SDS), State-Trait Anxiety Inventory (STAI), Qi, Blood, Fluid, Oriental Medicine

\section{Introduction}

When occidental medicine is ineffective for psychosomatic or psychiatric diseases, classification of the diseases according to the oriental concepts might be helpful for proper treatment. There have been many reports in which depressive state cases were attributed to visceral patterns such as spleen deficiency (Wang, Wang, Qiao, \& Li, 2009; Yuasa, 1999), liver blood deficiency (Yang \& Zhi, 1994), lung deficiency (Yuasa, 1999) and heart deficiency (Chan, Briscomb, Waterhouse, \& Cannaby, 2002). Apart from visceral patterns, the concept of Qi-bloodfluid patterns is also helpful, since there are many specific Chinese herbal extract preparations corresponding to specific combination of a few Qi-blood-fluid patterns. Qi represents vital energy, which is necessary for organ functions, production of kidney yang, and immune function. Qi abnormalities are divided into Qi-deficiency, Qi-stagnation, and Qi-flowback (K. Terasawa, 2004). Qi-deficiency is congenital or attributed to anorexia or excessive fatigue. Qi-stagnation results from im- paired intra- and extra-channel circulation of Qi. Qi-flowback is an adverse current of efferent or downward current of Qi. Representative blood patterns are blood deficiency resulting from reduced production or excessive consumption of blood, and blood stagnation resulting from cold, traumatic circulatory failure, or stasis (K. Terasawa, 2004).

There have been no reports in which the extents of Qi-blood-fluid patterns were calculated in patients with psychiatric or psychosomatic disorders. However, there have been some reports that depression or some anxiety disorder was treated with herbal extract preparations associated with Qiblood-fluid patterns. Thus, it was hypothesized that associations between some single Qi-blood-fluid pattern or some characteristic combination of Qi-blood-fluid patterns and each psychiatric or psychosomatic disorders such as depression, anxiety disorders etc. would be revealed by quantifying the extent of Qi-blood-fluid patterns of all the subjects. In order to quantify the extent of Qi-blood-fluid patterns, a scoring system (Appendix 1) was developed by Tera- sawa et al. (Katsutoshi Terasawa, 
1990; K. Terasawa, 2004). For example, the diagnostic criterion, Qi-deficiency score, was devised as follows. First, the author Terasawa synthetically observed subjective symptoms and objective findings of the patients. The severity of Qi-deficiency, assessed by the observation was graded to four levels by the author's empirical knowledge. The grade was set as "overall scale". Second, the prevalence of the symptoms and signs reported to be related to Qi-deficiency in the previous studies was ordered to four levels. The attributable proportion of prevalence for each symptom or sign to the overall scale was estimated by the multiple regression analysis, and accordingly constituted as Qi-deficiency score. In addition, Qi-stagnation, Qi-flowback, blood deficiency, blood stagnation, and fluid retention scores are calculated according to this system.

Although this scoring system was not subject to indefectible validation by a third person, it is the first and the only approach to quantify Qi-blood-fluid patterns. Thus, we considered it would be useful to calculate the extent of Qi-blood-fluid patterns of all the subjects and investigated the association between the extent of Qi-blood-fluid patterns and the major representative diseases at a psychosomatic clinic, including depression, anxiety disorder, eating disorder, and somatoform disorder according to Diagnostic and Statistical Manual of Mental Disorders 4th Edition (DSM-IV). In the present study, the Qi-blood-fluid pattern scores were calculated based on the chief complaints and answers to questionnaires according to Terasawa's Qi-blood-fluid scoring system for each patient who consulted the psychosomatic clinic at the tertiary medical care center. The associations between the Qi-blood-fluid patterns and indices of depressive state, i.e., Self-rating Depression Scale (SDS) (Zung, Richards, \& Short, 1965) scores, or indices of anxiety state, i.e., State-trait Anxiety Inventory (STAI) scores were also investigated.

\section{Materials and Methods}

\section{Subjects}

The subjects of this study were outpatients who visited for the first time during the period from June 2000 to March 2001 the clinic of the Department of Psychosomatic Medicine in Kyushu University Hospital, a tertiary medical care center. This department provides western psychosomatic and psychiatric medicine. Approximately half of them were walk-in patients and the others were referrals. After preexamination by the physician in order to make a provisional diagnosis, the patients were assigned to the attending physicians and given a complete checkup. Subjects with organic neurological disorders, endocrine disorders, neoplasm, vascular disorders, infection, degenerative diseases, or traumatism were excluded from the analyses. Subjects whose diagnoses could not be identified, whose chief complaints were not described, or who left more than 21 questions on the KMI questionnaire unanswered, were also excluded. Thus, 579 subjects remained among all the 914 outpatients. All of the 579 participant patients gave written informed consent for the relevant medical examinations. The protocol of the present survey was approved by the Research Ethics Committee of Graduate School of Medical Sciences, Kyushu University.

\section{Assessment of Symptoms}

At the preliminary examination, questionnaires and an interview were conducted with each outpatient, who gave written consent. All the patients were asked to describe up to three chief complaints. The questionnaires included the Kyudai Medical Index (KMI), a modification of the Cornell Medical Index-Health Questionnaire (Matsuoka, 1990), SDS, and STAI. Subjective psychiatric symptoms associated with depressive disorders were assessed by the SDS questionnaire. The state anxiety and trait anxiety were assessed by STAI-I and STAI-II, respectively, which were parts of STAI (Spielberger, Gorsuch, $\&$ Lushene, 1970). The reliability and validity of the SDS for testing depressive state have been proven (Zung et al., 1965). The cutoff score for screening depression has been reported to 40 points (Zung et al., 1965). The reliability and validity of Japanese version of STAI for testing anxiety has been proven, whose Cronbach's coefficient alpha was as much as 0.92 (Nakasato, 1990). The KMI consists of 102 yes or no questions, which include two comprehensive questions, two questions about past history, two questions about family history, 50 questions about psychiatric symptoms, and 46 questions about somatic symptoms. It has been reported that Category B and C scores in KMI could distinguish healthy controls from patients with psychosomatic disorders and those with neurotic disorders. It could also distinguish patients with neurotic disorders from those with psychosomatic disorders (Matsuoka, 1990).

\section{Evaluation of Symptoms and Signs in Oriental Syndromes}

The oriental syndromes derived from Qi, blood or fluid in each patient were evaluated according to Terasawa's Qi-bloodfluid scoring system for the diagnosis of Qi-deficiency, Qistagnation, Qi-flowback, blood stagnation, blood deficiency, and fluid retention (Katsutoshi Terasawa, 1990; K. Terasawa, 2004). For example, the B-42 question in the KMI, "Do you enjoy nothing and are you depressed?" was regarded as a depressive mood, which corresponds to 18 points for Qi-stagnation in Terasawa's system. As a whole, 8 of 14 factors in Qideficiency criteria, 11 of 12 factors in Qi-stagnation criteria, 11 of 12 factors in Qi-flowback criteria, 8 of 12 factors in blood deficiency criteria, and 11 of 18 factors in the fluid retention criteria corresponded to some of the chief complaints or questions in the KMI of all the subjects. However, among 17 blood stagnation factors, only one corresponded to any of the chief complaints or KMI questions. Therefore, blood stagnation scores were not calculated. Among the 102 questions of the KMI, 40 corresponded to factors of Terasawa's scoring system. The kinds of chief complaints reached 183. Among them, 58 were included in Terasawa's scoring system. The correspondence between symptoms described as chief complaints or in the KMI, and terms related to Qi-blood-fluid patterns are shown in Appendix 2 and 3, respectively. Some symptoms were inquired about twice using different words. Some symptoms corresponded to two Qi-blood-fluid patterns. In Terasawa's scoring system, a full point count is given when the symptom or sign is apparent and half the points are scored when it is slight. Therefore, full scores were assigned to the chief complaints corresponding to Terasawa's criteria, while symptoms which were not described as the chief complaints but described in the KMI questionnaire were regarded to be slight and half scores were assigned to them.

\section{Statistics}

All analyses were performed using Stata version 10.1 (Stata Corp., College Station, Texas, USA). Kolmogorov Smirnov's statistic was calculated to evaluate the normality of the distribution. Sexual differences in the proportion of diagnoses were tested by the chi-square test for independence. In order to com- 
pare the means of continuous variables of the two groups, Welch's $t$ test was used.

Multiple logistic regression analyses were performed to estimate the association between the prevalence of occidental diagnoses and Qi-deficiency, Qi-stagnation, Qi-flowback, blood deficiency, and fluid retention, as dummy variables for the nearest quartiles, adjusted for age ( $<40$ or $40+$ years old). To obtain the correlation between two continuous variables, Spearman's rank correlation coefficients were estimated. A two-sided $\mathrm{p}<0.05$ was considered to be statistically significant.

\section{Results}

\section{Demographic and Occidental Status}

The demographic and occidental aspects of the subjects are shown in Table 1. The continuous variables are shown as mean \pm standard deviation. The mean age of the subjects was 36.4 years old without significant sexual difference.

The average scores in SDS, STAI-I and STAI II were 50.6 points, 54.8 points and 55.3 points per 80 points, respectively.

In KMI, the average score for category $\mathrm{B}$ questions, i.e., questions assessing psychiatric symptoms, were 23.3 points per 50 points. The average score for the somatic symptoms categorized as KMI C questions was 15.4 per 47 points. These are close to those of patients with neurotic disorders in the previous study (Matsuoka, 1990).

The numbers of patients with each disorder and their percentage are shown. The prevalence of mood disorders, anxiety disorders, eating disorders, somatoform disorders, and irritable bowel syndrome was $54.2 \%, 19.9 \%, 10.9 \%, 7.6 \%$, and $6.6 \%$, respectively. The total numbers of patients with subclassification diseases did not equal the number in each corresponding major division due to overlapping.

\section{Oriental Status}

In order to investigate the extent of Qi-blood-fluid patterns according to sex and age, analysis of covariance was performed. As shown in Table 2, Qi-flowback, blood deficiency and fluid retention were significantly higher in females. Age was negatively correlated with fluid retention scores.

\section{Correlations among Qi-Blood-Fluid Patterns (Figure 1)}

Since Kolmogorov Smirnov's value showed skewed distribution of the fluid retention scores, Spearman's rank correlation coefficients were calculated among Qi-blood-fluid patterns by sex. Strong correlation coefficients $(>0.5)$ were observed between Qi-deficiency and Qi-stagnation in males and between blood deficiency and fluid retention in each sex. In females, the association between the scores was weak on the whole except for a strong association between blood deficiency and fluid retention. Relatively strong associations were observed among Qi-flowback, blood deficiency and fluid retention, between Qi-deficiency and Qi-stagnation, and between Qi-stagnation and Qi-flowback in females.

\section{Associations between Qi-Blood-Fluid Patterns and Occidental Diagnoses}

To investigate the association between oriental diagnoses as given above and occidental diagnoses according to major divisions as given by the attending physicians, multiple logistic regression analyses were conducted by sex. The adjusted odds ratio (OR) and its $95 \%$ confidence interval (CI) are shown in Table 3. In the same way, the association between oriental diagnoses according to minor divisions was also investigated and shown in Table 4. Although not shown in the tables, the categorizing age had almost no effect on the results.

Higher Qi-deficiency and Qi-stagnation were significantly associated with mood disorders and major depression in both sexes. On the other hand, Qi-deficiency scores were inversely associated with "depressive disorder not otherwise specified" in both sexes. The association between dysthymic disorder and the Qi-blood-fluid patterns was not statistically significant, whose data are not shown.

In anxiety disorders, the results were different between females and males. In females, Qi-flowback was associated with all anxiety disorders as shown in Table 3 and generalized anxiety disorder as shown in Table 4. In males, only blood deficiency was associated with generalized anxiety disorder as shown in Table 4.

Furthermore, fluid retention in females was associated with eating disorders as shown in Table 3. Qi-flowback and blood deficiency in females were associated with somatoform disorders. On the other hand, fluid retention in females was inversely associated with somatoform disorders.

Associations between somatic diseases and Qi-blood-fluid patterns were also investigated. As the only positive result, extremely high Qi-flowback in females was associated with irritable bowel syndrome, as shown in Table 4.

\section{Associations between Qi-Blood-Fluid Patterns and Psychological Questionnaire scores (Table 5)}

In order to investigate the oriental aspect of psychological defects, multiple logistic regression analysis was performed. The dependent variable was SDS, State or Trait anxiety scores. The explanatory variables were Qi-deficiency, Qi-stagnation, Qi-flowback, blood deficiency, and fluid retention.

In STAI scores, the results were different between females and males. In females, any Qi-pattern was associated with either high State or Trait anxiety scores. On the other hand, only high Qi-deficiency among the Qi-patterns was associated with high STAI scores in males. Instead, blood deficiency was associated with high State anxiety scores in males.

All the Qi-patterns in both sexes except for Qi-flowback in males and blood deficiency in males were associated with high SDS scores.

\section{Discussion}

The average score for psychiatric symptoms categorized as KMI B questions in the KMI was 23.3/50, which was higher than the average reported previously for normal subjects, $9.5 / 50$, and those with psychosomatic diseases, 18.8/50. However, it was lower than that of patients with neurosis, 24.4/50 (Matsuoka, 1990). The average score for the somatic symptoms categorized as KMI C questions was $15.4 / 47$, which was higher than that of normal subjects, 5.1/47, that of patients with psychosomatic diseases, $14.8 / 47$, and lower than that of neurosis, $15.6 / 47$, in the previous report (Matsuoka, 1990). Thus, the subjects of the present study were considered to range between psychosomatic and neurotic.

The present study indicates that blood deficiency and fluid retention scores were higher in females than males. The correlation coefficients were more than 0.5 between Qi-deficiency 
Table 1.

Demographic data on subjects.

\begin{tabular}{|c|c|c|c|c|c|c|}
\hline & \multicolumn{2}{|c|}{ Female $(n=364)$} & \multicolumn{2}{|c|}{ Male $(n=215)$} & \multicolumn{2}{|c|}{ Total $(\mathrm{n}=579)$} \\
\hline Age & \multicolumn{2}{|c|}{$36.5 \pm 17.0$} & \multicolumn{2}{|c|}{$36.3 \pm 16.7$} & \multicolumn{2}{|c|}{$36.4 \pm 16.9$} \\
\hline STAI-I (points) & \multicolumn{2}{|c|}{$55.4 \pm 11.2$} & \multicolumn{2}{|c|}{$53.7 \pm 11.8$} & \multicolumn{2}{|c|}{$54.8 \pm 11.5$} \\
\hline STAI-II (points) & \multicolumn{2}{|c|}{$56.1 \pm 11.7$} & \multicolumn{2}{|c|}{$53.9 \pm 12.3$} & \multicolumn{2}{|c|}{$55.3 \pm 12.0$} \\
\hline KMI category B questions (points) & \multicolumn{2}{|c|}{$23.5 \pm 10.3$} & \multicolumn{2}{|c|}{$22.9 \pm 10.6$} & \multicolumn{2}{|c|}{$23.3 \pm 10.4$} \\
\hline Mood disorders & 205 & $(56.3)$ & 109 & $(50.7)$ & 314 & $(54.2)$ \\
\hline Major depression & 158 & $(43.4)$ & 79 & $(36.7)$ & 237 & $(46.8)$ \\
\hline Dysthymic disorder & 23 & $(6.3)$ & 8 & $(3.7)$ & 31 & $(5.4)$ \\
\hline Anxiety disorders & 71 & $(19.5)$ & 44 & $(20.5)$ & 115 & $(19.9)$ \\
\hline Generalized anxiety disorder & 35 & $(9.6)$ & 22 & $(10.2)$ & 57 & $(9.8)$ \\
\hline Specific phobia & 3 & $(0.8)$ & 2 & $(0.9)$ & 5 & $(0.9)$ \\
\hline Panic disorder & 24 & $(6.6)$ & 11 & $(5.1)$ & 35 & $(6.0)$ \\
\hline Posttraumatic stress disorder & 1 & $(0.3)$ & 2 & $(0.9)$ & 3 & $(0.5)$ \\
\hline Social anxiety disorder & 4 & $(1.1)$ & 8 & $(3.7)$ & 12 & $(2.1)$ \\
\hline Obsessive compulsive disorder & 5 & (1.4) & 1 & $(0.5)$ & 6 & $(1.0)$ \\
\hline Eating disorders & 57 & $(15.7)$ & 6 & $(2.8)$ & 63 & $(10.9)$ \\
\hline Bulimia Nervosa & 27 & (7.4) & 1 & $(0.5)$ & 28 & $(4.8)$ \\
\hline Eating disorder not otherwise specified & 13 & (3.6) & 2 & $(0.9)$ & 15 & $(2.6)$ \\
\hline Somatoform disorders & 28 & (7.7) & 16 & (7.4) & 44 & (7.6) \\
\hline Conversion disorder & 4 & $(1.1)$ & 0 & $(0.0)$ & 4 & $(0.7)$ \\
\hline Hypochondriasis & 3 & $(0.8)$ & 3 & $(1.4)$ & 6 & $(1.0)$ \\
\hline Somatization disorder & 1 & $(0.3)$ & 0 & $(0.0)$ & 1 & $(0.2)$ \\
\hline Pain disorder & 15 & $(4.1)$ & 10 & $(4.7)$ & 25 & $(4.3)$ \\
\hline Somatoform disorder not otherwise specified & 4 & $(1.1)$ & 2 & $(0.9)$ & 6 & $(1.0)$ \\
\hline Irritable bowel syndrome & 20 & $(5.5)$ & 18 & $(8.4)$ & 38 & (6.6) \\
\hline Nonulcer dyspepsia & 15 & $(4.1)$ & 9 & $(4.2)$ & 24 & $(4.1)$ \\
\hline Bronchial asthma & 7 & $(1.9)$ & 2 & $(0.9)$ & 9 & $(1.6)$ \\
\hline Hypertension & 9 & $(2.5)$ & 4 & (1.9) & 13 & $(2.2)$ \\
\hline Hyperventilation syndrome & 10 & $(2.7)$ & 2 & $(0.9)$ & 12 & $(2.1)$ \\
\hline Dystonia & 5 & (1.4) & 3 & (1.4) & 8 & (1.4) \\
\hline Schizophrenia & 3 & $(0.8)$ & 5 & $(2.3)$ & 8 & $(1.4)$ \\
\hline Chronic fatigue syndrome & 1 & $(0.3)$ & 2 & $(0.9)$ & 3 & $(0.5)$ \\
\hline Dissociative disorder & 2 & $(0.5)$ & 0 & $(0.0)$ & 2 & $(0.3)$ \\
\hline
\end{tabular}

The numbers of patients are shown. Prevalence rates are expressed as percentages and enclosed within parentheses according to sex. 
Table 2 .

Qi-blood-fluid pattern scores according to terasawa's criteria.

\begin{tabular}{|c|c|c|c|c|}
\hline & Total (579 subjects) $)^{1)}$ & Female (364 subjects) ${ }^{1)}$ & Male (215 subjects $)^{1)}$ & Sexual difference \\
\hline Qi-deficiency & $14.3 \pm 6.7$ & $14.5 \pm 6.4$ & $13.8 \pm 7.1$ & $p=0.17$ \\
\hline Qi-stagnation & $17.4 \pm 10.5$ & $17.6 \pm 10.4$ & $17.1 \pm 10.6$ & $p=0.59$ \\
\hline Qi-flowback & $14.2 \pm 8.0$ & $14.8 \pm 8.0$ & $13.2 \pm 8.0$ & $p=0.02$ \\
\hline Blood deficiency & $12.9 \pm 6.7$ & $13.3 \pm 6.6$ & $12.1 \pm 6.7$ & $p=0.04$ \\
\hline Fluid retention & $6.4 \pm 5.0$ & $7.0 \pm 5.2$ & $5.2 \pm 4.3$ & $p<0.0001$ \\
\hline
\end{tabular}

${ }^{1)}$ Expressed as mean \pm standard deviation

Table 3.

Association between occidental diagnoses according to major division and qi-blood-fluid patterns.

\begin{tabular}{|c|c|c|c|c|c|c|c|}
\hline \multirow[b]{2}{*}{$\begin{array}{l}\text { Dependent } \\
\text { variable }\end{array}$} & \multirow[b]{2}{*}{$\begin{array}{l}\text { Explanatory } \\
\text { variable }\end{array}$} & \multicolumn{3}{|c|}{ Females } & \multicolumn{3}{|c|}{ Males } \\
\hline & & $\begin{array}{l}\text { Range of the } \\
\text { category }\end{array}$ & $\begin{array}{c}\text { Odds ratio } \\
(95 \% \text { confidence interval })\end{array}$ & $p$ & $\begin{array}{l}\text { Range of the } \\
\text { category }\end{array}$ & $\begin{array}{c}\text { Odds ratio } \\
(95 \% \text { confidence interval })\end{array}$ & $p$ \\
\hline & & $0-10$ & 1 (referent) & & $0-9$ & 1 (referent) & \\
\hline & & $11-16$ & $1.41(0.71-2.82)$ & 0.33 & $10-14$ & $1.85(0.72-4.77)$ & 0.20 \\
\hline & Qi-deficiency & $17-19$ & $2.26(1.14-4.51)$ & 0.02 & $15-19$ & $0.92(0.35-2.40)$ & 0.86 \\
\hline & & $20-31$ & $3.39(1.50-7.65)$ & $<0.01$ & $20-30$ & $6.36(1.94-20.87)$ & $<0.01$ \\
\hline & & & Trend $\mathrm{P}<0.01$ & & & Trend $\mathrm{P}=0.03$ & \\
\hline & & $0-9$ & 1 (referent) & & $0-8$ & 1 (referent) & \\
\hline & Qi-stagnation & $18-26$ & $3.90(1.97-7.73)$ & $<0.001$ & $18-26$ & $2.93(1.10-7.82)$ & 0.03 \\
\hline & & $27-46$ & $5.42(2.37-12.41)$ & $<0.001$ & $27-40$ & $4.89(1.53-15.66)$ & $<0.01$ \\
\hline & & & Trend $\mathrm{P}<0.001$ & & & Trend $\mathrm{P}<0.01$ & \\
\hline & & $0-8$ & 1 (referent) & & $0-7$ & 1 (referent) & \\
\hline & & $9-15$ & $1.40(0.73-2.68)$ & 0.31 & $8-13$ & $1.26(0.51-3.10)$ & 0.62 \\
\hline \multirow[t]{12}{*}{ Mood disorders } & Qi-flowback & $16-21$ & $1.65(0.81-3.36)$ & 0.17 & $14-19$ & $2.39(0.89-6.39)$ & 0.08 \\
\hline & & $22-37$ & $0.92(0.41-2.04)$ & 0.83 & $20-36$ & $1.71(0.56-5.21)$ & 0.34 \\
\hline & & & Trend $\mathrm{P}=1.00$ & & & Trend $\mathrm{P}=0.15$ & \\
\hline & & $10-13$ & $1.67(0.85-3.27)$ & 0.13 & $7-12$ & $0.83(0.35-1.96)$ & 0.67 \\
\hline & Blood deficiency & $14-18$ & $1.42(0.70-2.89)$ & 0.33 & $13-16$ & $0.40(0.14-1.10)$ & 0.07 \\
\hline & & $19-29$ & $1.17(0.53-2.54)$ & 0.70 & $17-28$ & $0.58(0.19-1.74)$ & 0.33 \\
\hline & & & Trend $\mathrm{P}=0.61$ & & & Trend $\mathrm{P}=0.30$ & \\
\hline & & $0-2.5$ & 1 (referent) & & $0-2.5$ & 1 (referent) & \\
\hline & & $3-6.5$ & $1.12(0.57-2.19)$ & 0.74 & $3-5$ & $1.14(0.49-2.63)$ & 0.76 \\
\hline & Fluid retention & $7-10.5$ & $1.31(0.61-2.81)$ & 0.49 & $5.5-7.5$ & $0.42(0.14-1.26)$ & 0.12 \\
\hline & & $11-19.5$ & $0.65(0.30-1.44)$ & 0.29 & $8-20.5$ & $2.11(0.81-5.53)$ & 0.13 \\
\hline & & & Trend $\mathrm{P}=0.32$ & & & Trend $\mathrm{P}=0.08$ & \\
\hline
\end{tabular}




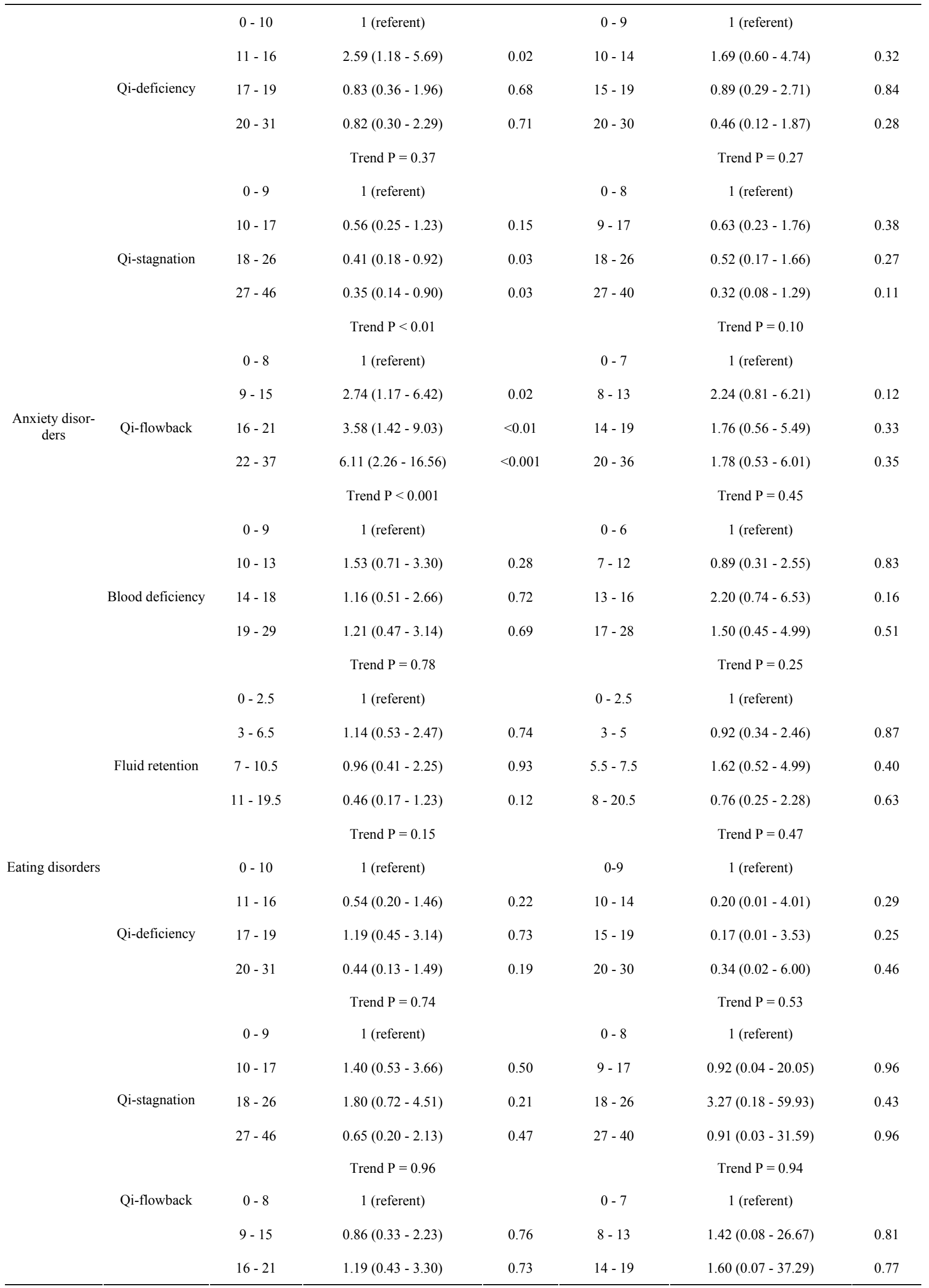




\begin{tabular}{|c|c|c|c|c|c|c|c|}
\hline & & $22-37$ & $0.65(0.21-2.01)$ & 0.45 & $20-36$ & $5.20(0.27-98.49)$ & 0.27 \\
\hline & & & Trend $\mathrm{P}=0.55$ & & & Trend $\mathrm{P}=0.22$ & \\
\hline & & $0-9$ & 1 (referent) & & $0-6$ & 1 (referent) & \\
\hline & & $10-13$ & $0.98(0.38-2.52)$ & 0.97 & $7-12$ & $0.95(0.05-19.40)$ & 0.97 \\
\hline & Blood deficiency & $14-18$ & $0.56(0.21-1.54)$ & 0.26 & $13-16$ & $1.67(0.08-34.72)$ & 0.74 \\
\hline & & $19-29$ & $0.36(0.13-1.06)$ & 0.06 & $17-28$ & $2.21(0.09-52.26)$ & 0.62 \\
\hline & & & Trend $\mathrm{P}=0.05$ & & & Trend $\mathrm{P}=0.64$ & \\
\hline & & $0-2.5$ & 1 (referent) & & $0-2.5$ & 1 (referent) & \\
\hline & & $3-6.5$ & $0.70(0.24-2.03)$ & 0.51 & $3-5$ & $0.39(0.03-5.38)$ & 0.48 \\
\hline & Fluid retention & $7-10.5$ & $2.28(0.79-6.57)$ & 0.13 & $5.5-7.5$ & $0.32(0.02-6.58)$ & 0.46 \\
\hline & & $11-19.5$ & $6.10(2.09-17.79)$ & $<0.001$ & $8-20.5$ & $0.97(0.09-10.92)$ & 0.98 \\
\hline & & & Trend $\mathrm{P}<0.001$ & & & Trend $\mathrm{P}=0.83$ & \\
\hline & & $0-10$ & 1 (referent) & & $0-9$ & 1 (referent) & \\
\hline & & $11-16$ & $0.16(0.04-0.69)$ & 0.01 & $10-14$ & $1.12(0.16-7.75)$ & 0.91 \\
\hline & Qi-deficiency & $17-19$ & $0.19(0.05-0.72)$ & 0.01 & $15-19$ & $2.40(0.42-13.85)$ & 0.33 \\
\hline & & $20-31$ & $0.23(0.05-1.13)$ & 0.07 & $20-30$ & $1.02(0.12-8.74)$ & 0.99 \\
\hline & & & Trend $\mathrm{P}<0.01$ & & & Trend $\mathrm{P}=0.68$ & \\
\hline & & $0-9$ & 1 (referent) & & $0-8$ & 1 (referent) & \\
\hline & & $10-17$ & $0.64(0.18-2.32)$ & 0.50 & $9-17$ & $1.21(0.16-9.41)$ & 0.85 \\
\hline & Qi-stagnation & $18-26$ & $0.48(0.12-1.93)$ & 0.30 & $18-26$ & $3.34(0.56-19.98)$ & 0.19 \\
\hline & & $27-46$ & $0.25(0.04-1.69)$ & 0.16 & $27-40$ & $5.07(0.62-41.22)$ & 0.13 \\
\hline & & & Trend $\mathrm{P}=0.16$ & & & Trend $\mathrm{P}=0.09$ & \\
\hline & & $0-8$ & 1 (referent) & & $0-7$ & 1 (referent) & \\
\hline & & $9-15$ & $2.72(0.76-9.71)$ & 0.12 & $8-13$ & $0.12(0.01-1.35)$ & 0.09 \\
\hline Somatoform & Qi-flowback & $16-21$ & $4.13(1.05-16.28)$ & 0.04 & $14-19$ & $1.01(0.19-5.24)$ & 0.99 \\
\hline & & $22-37$ & $7.65(1.51-38.84)$ & 0.01 & $20-36$ & $1.03(0.16-6.70)$ & 0.98 \\
\hline & & & Trend $\mathrm{P}=0.01$ & & & Trend $\mathrm{P}=0.43$ & \\
\hline & & $0-9$ & 1 (referent) & & $0-6$ & 1 (referent) & \\
\hline & & $10-13$ & $0.98(0.22-4.27)$ & 0.98 & $7-12$ & $0.86(0.20-3.79)$ & 0.84 \\
\hline & Blood deficiency & $14-18$ & $3.33(0.87-12.68)$ & 0.08 & $13-16$ & $0.73(0.11-4.79)$ & 0.74 \\
\hline & & $19-29$ & $25.49(4.82-134.67)$ & $<0.001$ & $17-28$ & $0.73(0.11-5.02)$ & 0.75 \\
\hline & & & Trend $\mathrm{P}<0.001$ & & & Trend $\mathrm{P}=1.00$ & \\
\hline & & $0-2.5$ & 1 (referent) & & $0-2.5$ & 1 (referent) & \\
\hline & & $3-6.5$ & $0.35(0.10-1.15)$ & 0.08 & $3-5$ & $0.40(0.09-1.81)$ & 0.23 \\
\hline & Fluid retention & $7-10.5$ & $0.05(0.01-0.27)$ & $<0.001$ & $5.5-7.5$ & $0.25(0.03-1.84)$ & 0.17 \\
\hline & & $11-19.5$ & $0.03(0.01-0.20)$ & $<0.001$ & $8-20.5$ & $0.08(0.01-0.90)$ & 0.04 \\
\hline & & & Trend $\mathrm{P}<0.001$ & & & Trend $\mathrm{P}=0.05$ & \\
\hline
\end{tabular}


Table 4.

Association between occidental diagnoses according to major division and Qi-blood-fluid patterns.

\begin{tabular}{|c|c|c|c|c|c|c|c|}
\hline \multirow[b]{2}{*}{$\begin{array}{l}\text { Dependent } \\
\text { variable }\end{array}$} & \multirow[b]{2}{*}{$\begin{array}{l}\text { Explanatory } \\
\text { variable }\end{array}$} & \multicolumn{3}{|c|}{ Females } & \multicolumn{3}{|c|}{ Males } \\
\hline & & $\begin{array}{l}\text { Range of the } \\
\text { category }\end{array}$ & $\begin{array}{c}\text { Odds ratio } \\
(95 \% \text { confidence interval) }\end{array}$ & $p$ & $\begin{array}{l}\text { Range of the } \\
\text { category }\end{array}$ & $\begin{array}{c}\text { Odds ratio } \\
(95 \% \text { confidence interval) }\end{array}$ & $p$ \\
\hline & & $0-10$ & 1 (referent) & & $0-9$ & 1 (referent) & \\
\hline & & $11-16$ & $3.17(1.32-7.60)$ & $<0.01$ & $10-14$ & $11.66(2.02-67.28)$ & $<0.01$ \\
\hline & Qi-deficiency & $17-19$ & $4.94(2.09-11.70)$ & $<0.001$ & $15-19$ & $8.08(1.48-44.31)$ & 0.02 \\
\hline & & $20-31$ & $8.43(3.21-22.17)$ & $<0.001$ & $20-30$ & $58.60(8.92-384.93)$ & $<0.001$ \\
\hline & & & Trend $\mathrm{P}<0.001$ & & & Trend $\mathrm{P}<0.001$ & \\
\hline & & $0-9$ & 1 (referent) & & $0-8$ & 1 (referent) & \\
\hline & & $10-17$ & $3.78(1.64-8.72)$ & $<0.01$ & $9-17$ & $2.55(0.62-10.50)$ & 0.19 \\
\hline & Qi-stagnation & $18-26$ & $5.60(2.45-12.79)$ & $<0.001$ & $18-26$ & $3.89(0.93-16.21)$ & 0.06 \\
\hline & & $27-46$ & $8.84(3.44-22.70)$ & $<0.001$ & $27-40$ & $8.70(1.94-39.07)$ & $<0.01$ \\
\hline & & & Trend $\mathrm{P}<0.001$ & & & Trend $\mathrm{P}=0.02$ & \\
\hline & & $0-8$ & 1 (referent) & & $0-7$ & 1 (referent) & \\
\hline & & $9-15$ & $1.31(0.60-2.85)$ & 0.50 & $8-13$ & $1.16(0.33-4.16)$ & 0.82 \\
\hline \multirow[t]{13}{*}{ Major depression } & Qi-flowback & $16-21$ & $1.73(0.75-3.95)$ & 0.20 & $14-19$ & $2.90(0.76-11.14)$ & 0.12 \\
\hline & & $22-37$ & $1.07(0.44-2.61)$ & 0.88 & $20-36$ & $1.54(0.37-6.50)$ & 0.55 \\
\hline & & & Trend $\mathrm{P}=0.82$ & & & Trend $\mathrm{P}=0.29$ & \\
\hline & & $0-9$ & 1 (referent) & & $0-6$ & 1 (referent) & \\
\hline & & $10-13$ & $1.59(0.73-3.44)$ & 0.24 & $7-12$ & $1.22(0.39-3.79)$ & 0.74 \\
\hline & Blood deficiency & $14-18$ & $1.08(0.47-2.47)$ & 0.85 & $13-16$ & $0.48(0.13-1.81)$ & 0.28 \\
\hline & & $19-29$ & $1.05(0.44-2.52)$ & 0.91 & $17-28$ & $0.82(0.21-3.17)$ & 0.77 \\
\hline & & & Trend $\mathrm{P}=0.98$ & & & Trend $\mathrm{P}=0.94$ & \\
\hline & & $0-2.5$ & 1 (referent) & & $0-2.5$ & 1 (referent) & \\
\hline & & $3-6.5$ & $0.67(0.31-1.48)$ & 0.33 & $3-5$ & $0.94(0.31-2.88)$ & 0.92 \\
\hline & Fluid retention & $7-10.5$ & $1.10(0.46-2.63)$ & 0.83 & $5.5-7.5$ & $0.47(0.13-1.73)$ & 0.26 \\
\hline & & $11-19.5$ & $0.63(0.26-1.53)$ & 0.31 & $8-20.5$ & $2.02(0.57-7.10)$ & 0.27 \\
\hline & & & Trend $\mathrm{P}=0.50$ & & & Trend $\mathrm{P}=0.12$ & \\
\hline \multirow[t]{6}{*}{$\begin{array}{l}\text { Depressive disorder } \\
\text { not otherwise specified }\end{array}$} & & $0-10$ & 1 (referent) & & $0-9$ & 1 (referent) & \\
\hline & & $11-16$ & $0.50(0.20-1.23)$ & 0.13 & $10-14$ & $0.80(0.25-2.54)$ & 0.71 \\
\hline & Qi-deficiency & $17-19$ & $0.35(0.13-0.94)$ & 0.04 & $15-19$ & $0.08(0.01-0.49)$ & $<0.01$ \\
\hline & & $20-31$ & $0.21(0.05-0.82)$ & 0.03 & $20-30$ & $0.05(0.00-0.51)$ & 0.01 \\
\hline & & & Trend $\mathrm{P}<0.01$ & & & Trend $\mathrm{P}<0.001$ & \\
\hline & Qi-stagnation & $0-9$ & 1 (referent) & & $0-8$ & 1 (referent) & \\
\hline
\end{tabular}




\begin{tabular}{|c|c|c|c|c|c|c|c|}
\hline & & $10-17$ & $0.66(0.25-1.77)$ & 0.41 & $9-17$ & $1.31(0.40-4.21)$ & 0.66 \\
\hline & & $18-26$ & $1.04(0.41-2.60)$ & 0.94 & $18-26$ & $2.57(0.69-9.59)$ & 0.16 \\
\hline & & $27-46$ & $0.34(0.08-1.43)$ & 0.14 & $27-40$ & $0.28(0.03-3.06)$ & 0.30 \\
\hline & & & Trend $\mathrm{P}=0.40$ & & & Trend $\mathrm{P}=0.66$ & \\
\hline & & $0-8$ & 1 (referent) & & $0-7$ & 1 (referent) & \\
\hline & & $9-15$ & $1.14(0.46-2.81)$ & 0.77 & $8-13$ & $1.94(0.61-6.15)$ & 0.26 \\
\hline & Qi-flowback & $16-21$ & $1.98(0.77-5.07)$ & 0.16 & $14-19$ & $1.84(0.50-6.84)$ & 0.36 \\
\hline & & $22-37$ & $0.49(0.12-2.03)$ & 0.33 & $20-36$ & $2.67(0.58-12.37)$ & 0.21 \\
\hline & & & Trend $\mathrm{P}=0.95$ & & & Trend $\mathrm{P}=0.13$ & \\
\hline & & $0-9$ & 1 (referent) & & $0-6$ & 1 (referent) & \\
\hline & & $10-13$ & $1.13(0.44-2.86)$ & 0.80 & $7-12$ & $0.67(0.21-2.10)$ & 0.49 \\
\hline & Blood deficiency & $14-18$ & $1.62(0.64-4.13)$ & 0.31 & $13-16$ & $0.78(0.21-2.93)$ & 0.72 \\
\hline & & $19-29$ & $1.06(0.32-3.49)$ & 0.93 & $17-28$ & $0.28(0.05-1.52)$ & 0.14 \\
\hline & & & Trend $\mathrm{P}=0.59$ & & & Trend $\mathrm{P}=0.22$ & \\
\hline & & $0-2.5$ & 1 (referent) & & $0-2.5$ & 1 (referent) & \\
\hline & & $3-6.5$ & $2.55(1.03-6.30)$ & 0.04 & $3-5$ & $1.28(0.42-3.95)$ & 0.66 \\
\hline & Fluid retention & $7-10.5$ & $1.88(0.69-5.13)$ & 0.22 & $5.5-7.5$ & $0.19(0.02-1.93)$ & 0.16 \\
\hline & & $11-19.5$ & $0.28(0.05-1.45)$ & 0.13 & $8-20.5$ & $2.14(0.62-7.38)$ & 0.23 \\
\hline & & & Trend $\mathrm{P}=0.23$ & & & Trend $\mathrm{P}=0.23$ & \\
\hline $\begin{array}{l}\text { Generalized anxiety } \\
\text { disorder }\end{array}$ & & $0-10$ & 1 (referent) & & $0-9$ & 1 (referent) & \\
\hline & & $11-16$ & $2.17(0.78-6.01)$ & 0.14 & $10-14$ & $0.91(0.25-3.35)$ & 0.89 \\
\hline & Qi-deficiency & $17-19$ & $0.32(0.09-1.21)$ & 0.09 & $15-19$ & $0.52(0.12-2.20)$ & 0.37 \\
\hline & & $20-31$ & $1.02(0.29-3.59)$ & 0.98 & $20-30$ & $0.20(0.03-1.40)$ & 0.10 \\
\hline & & & Trend $\mathrm{P}=0.29$ & & & Trend $\mathrm{P}=0.10$ & \\
\hline & & $0-9$ & 1 (referent) & & $0-8$ & 1 (referent) & \\
\hline & & $10-17$ & $0.47(0.16-1.39)$ & 0.17 & $9-17$ & $1.26(0.31-5.05)$ & 0.75 \\
\hline & Qi-stagnation & $18-26$ & $0.44(0.14-1.34)$ & 0.15 & $18-26$ & $0.87(0.17-4.40)$ & 0.87 \\
\hline & & $27-46$ & $0.48(0.14-1.67)$ & 0.25 & $27-40$ & $0.98(0.16-6.08)$ & 0.98 \\
\hline & & & Trend $\mathrm{P}=0.07$ & & & Trend $\mathrm{P}=0.89$ & \\
\hline & & $0-8$ & 1 (referent) & & $0-7$ & 1 (referent) & \\
\hline & & $9-15$ & $1.73(0.55-5.44)$ & 0.35 & $8-13$ & $3.21(0.78-13.24)$ & 0.11 \\
\hline & Qi-flowback & $16-21$ & $4.23(1.32-13.59)$ & 0.02 & $14-19$ & $1.73(0.34-8.80)$ & 0.51 \\
\hline & & $22-37$ & $4.44(1.11-17.74)$ & 0.03 & $20-36$ & $1.79(0.35-9.17)$ & 0.49 \\
\hline & & & Trend $\mathrm{P}=0.01$ & & & Trend $\mathrm{P}=0.90$ & \\
\hline & Blood deficiency & $0-9$ & 1 (referent) & & $0-6$ & 1 (referent) & \\
\hline
\end{tabular}




\begin{tabular}{|c|c|c|c|c|c|c|c|}
\hline & & $10-13$ & $0.71(0.23-2.23)$ & 0.56 & $7-12$ & $1.50(0.30-7.50)$ & 0.62 \\
\hline & & $14-18$ & $1.10(0.37-3.27)$ & 0.86 & $13-16$ & $5.63(1.14-27.84)$ & 0.03 \\
\hline & & $19-29$ & $1.12(0.30-4.16)$ & 0.87 & $17-28$ & $6.73(1.21-37.40)$ & 0.03 \\
\hline & & & Trend $\mathrm{P}=0.71$ & & & Trend $\mathrm{P}=0.02$ & \\
\hline & & $0-2.5$ & 1 (referent) & & $0-2.5$ & 1 (referent) & \\
\hline & & $3-6.5$ & $0.36(0.10-1.25)$ & 0.11 & $3-5$ & $0.64(0.17-2.36)$ & 0.50 \\
\hline & Fluid retention & $7-10.5$ & $0.95(0.33-2.79)$ & 0.93 & $5.5-7.5$ & $0.89(0.22-3.70)$ & 0.88 \\
\hline & & $11-19.5$ & $0.50(0.14-1.81)$ & 0.29 & $8-20.5$ & $0.17(0.03-1.02)$ & 0.05 \\
\hline & & & Trend $\mathrm{P}=0.48$ & & & Trend $\mathrm{P}=0.03$ & \\
\hline & & $0-10$ & 1 (referent) & & $0-9$ & 1 (referent) & \\
\hline & & $11-16$ & $0.90(0.17-4.83)$ & 0.90 & $10-14$ & $1.17(0.21-6.65)$ & 0.86 \\
\hline & Qi-deficiency & $17-19$ & $1.55(0.32-7.56)$ & 0.59 & $15-19$ & $1.25(0.21-7.39)$ & 0.80 \\
\hline & & $20-31$ & $2.09(0.38-11.53)$ & 0.40 & $20-30$ & $2.88(0.42-19.67)$ & 0.28 \\
\hline & & & Trend $\mathrm{P}=0.28$ & & & Trend $\mathrm{P}=0.30$ & \\
\hline & & $0-9$ & 1 (referent) & & $0-8$ & 1 (referent) & \\
\hline & & $10-17$ & $0.62(0.12-3.26)$ & 0.57 & $9-17$ & $0.25(0.04-1.63)$ & 0.15 \\
\hline & Qi-stagnation & $18-26$ & $0.93(0.21-4.09)$ & 0.92 & $18-26$ & $0.77(0.15-3.91)$ & 0.76 \\
\hline & & $27-46$ & $1.51(0.32-7.08)$ & 0.60 & $27-40$ & $0.43(0.06-3.05)$ & 0.40 \\
\hline & & & Trend $\mathrm{P}=0.41$ & & & Trend $\mathrm{P}=0.63$ & \\
\hline & & $0-8$ & 1 (referent) & & $0-7$ & 1 (referent) & \\
\hline & & $9-15$ & $1.18(0.24-5.92)$ & 0.84 & $8-13$ & $1.86(0.38-9.25)$ & 0.45 \\
\hline Irritable bowel & Qi-flowback & $16-21$ & $1.84(0.37-9.27)$ & 0.46 & $14-19$ & $1.31(0.22-7.94)$ & 0.77 \\
\hline & & $22-37$ & $5.14(1.03-25.59)$ & 0.05 & $20-36$ & $2.11(0.31-14.53)$ & 0.45 \\
\hline & & & Trend $\mathrm{P}=0.03$ & & & Trend $\mathrm{P}=0.54$ & \\
\hline & & $0-9$ & 1 (referent) & & $0-6$ & 1 (referent) & \\
\hline & & $10-13$ & $0.34(0.08-1.38)$ & 0.13 & $7-12$ & $0.82(0.19-3.50)$ & 0.79 \\
\hline & Blood deficiency & $14-18$ & $0.35(0.08-1.50)$ & 0.16 & $13-16$ & $0.52(0.10-2.60)$ & 0.42 \\
\hline & & $19-29$ & $0.27(0.05-1.45)$ & 0.13 & $17-28$ & $0.15(0.02-1.21)$ & 0.08 \\
\hline & & & Trend $\mathrm{P}=0.10$ & & & Trend $\mathrm{P}=0.13$ & \\
\hline & & $0-2.5$ & 1 (referent) & & $0-2.5$ & 1 (referent) & \\
\hline & & $3-6.5$ & $6.74(1.34-33.87)$ & 0.02 & $3-5$ & $0.53(0.09-3.08)$ & 0.48 \\
\hline & Fluid retention & $7-10.5$ & $1.18(0.14-9.90)$ & 0.88 & $5.5-7.5$ & $2.81(0.56-14.13)$ & 0.21 \\
\hline & & $11-19.5$ & $2.04(0.27-15.48)$ & 0.49 & $8-20.5$ & $1.92(0.41-9.09)$ & 0.41 \\
\hline & & & Trend $\mathrm{P}=0.63$ & & & Trend $\mathrm{P}=0.38$ & \\
\hline
\end{tabular}


Table 5 .

Association between SDS, state anxiety, trait anxiety scores and qi-blood-fluid patterns in females (a) and males (b).

(a)

\begin{tabular}{|c|c|c|c|c|c|c|c|c|c|}
\hline & \multicolumn{3}{|c|}{ State anxiety } & \multicolumn{3}{|c|}{ Trait anxiety } & \multicolumn{3}{|c|}{ SDS } \\
\hline & $\begin{array}{l}\text { Range of the } \\
\text { category }\end{array}$ & $\begin{array}{c}\text { Odds ratio }(95 \% \\
\text { confidence interval) }\end{array}$ & $p$ & $\begin{array}{l}\text { Range of the } \\
\text { category }\end{array}$ & $\begin{array}{c}\text { Odds ratio ( } 95 \% \\
\text { confidence interval) }\end{array}$ & $p$ & $\begin{array}{l}\text { Range of the } \\
\text { category }\end{array}$ & $\begin{array}{c}\text { Odds ratio }(95 \% \\
\text { confidence interval) }\end{array}$ & $p$ \\
\hline \multirow{5}{*}{ Qi-deficiency } & $0-10$ & 1 (referent) & & $0-10$ & 1 (referent) & & $0-10$ & 1 (referent) & \\
\hline & $11-16$ & $4.31(1.17-7.46)$ & $<0.01$ & $11-16$ & $5.37(2.09-8.65)$ & $<0.01$ & $11-16$ & $2.50(-0.10-5.10)$ & 0.06 \\
\hline & $17-19$ & $4.45(1.27-7.64)$ & $<0.01$ & $17-19$ & $8.88(5.57-12.18)$ & $<0.001$ & $17-19$ & $5.47(2.90-8.04)$ & $<0.001$ \\
\hline & $20-31$ & $3.38(-0.18-6.95)$ & 0.06 & $20-31$ & $9.47(5.77-13.17)$ & $<0.001$ & $20-31$ & $6.03(3.19-8.87)$ & $<0.001$ \\
\hline & & Trend $\mathrm{P}=0.02$ & & & Trend $\mathrm{P}<0.001$ & & & Trend $\mathrm{P}<0.001$ & \\
\hline \multirow{4}{*}{ Qi-stagnation } & $0-9$ & 1 (referent) & & $0-9$ & 1 (referent) & & $0-9$ & 1 (referent) & \\
\hline & $18-26$ & $6.66(3.48-9.83)$ & $<0.001$ & $18-26$ & $4.27(0.97-7.57)$ & 0.01 & $18-26$ & $7.82(5.25-10.38)$ & $<0.001$ \\
\hline & $27-46$ & $8.55(4.88-12.22)$ & $<0.001$ & $27-46$ & $3.49(-0.34-7.31)$ & 0.07 & $27-46$ & $9.35(6.41-12.29)$ & $<0.001$ \\
\hline & & Trend $\mathrm{P}<0.001$ & & & Trend $\mathrm{P}=0.07$ & & & Trend $\mathrm{P}<0.001$ & \\
\hline \multirow{4}{*}{ Qi-flowback } & $0-8$ & 1 (referent) & & $0-8$ & 1 (referent) & & $0-8$ & 1 (referent) & \\
\hline & $9-15$ & $3.49(0.52-6.46)$ & 0.02 & $9-15$ & $4.01(0.88-7.14)$ & 0.01 & $9-15$ & $2.93(0.48-5.38)$ & 0.02 \\
\hline & $16-21$ & $3.94(0.67-7.22)$ & 0.02 & $16-21$ & $5.74(2.29-9.19)$ & $<0.01$ & $16-21$ & $5.08(2.40-7.75)$ & $<0.001$ \\
\hline & $22-37$ & $4.85(1.17-8.54)$ & $<0.01$ & $22-37$ & $6.67(2.70-10.65)$ & $<0.01$ & $22-37$ & $6.04(3.05-9.02)$ & $<0.001$ \\
\hline \multirow{4}{*}{ Blood deficiency } & $10-13$ & $1.43(-1.58-4.44)$ & 0.35 & $10-13$ & $1.67(-1.54-4.88)$ & 0.31 & $10-13$ & $2.02(-0.41-4.45)$ & 0.1 \\
\hline & $14-18$ & $1.32(-1.87-4.52)$ & 0.42 & $14-18$ & $1.35(-1.94-4.64)$ & 0.42 & $14-18$ & $1.85(-0.73-4.42)$ & 0.16 \\
\hline & $19-29$ & $1.89(-1.73-5.52)$ & 0.31 & $19-29$ & $1.13(-2.60-4.85)$ & 0.55 & $19-29$ & $2.83(-0.04-5.71)$ & 0.05 \\
\hline & & Trend $\mathrm{P}=0.29$ & & & Trend $\mathrm{P}=0.49$ & & & Trend $\mathrm{P}=0.05$ & \\
\hline \multirow{5}{*}{ Fluid retention } & $0-2.5$ & 1 (referent) & & $0-2.5$ & 1 (referent) & & $0-2.5$ & 1 (referent) & \\
\hline & $3-6.5$ & $-0.12(-3.15-2.90)$ & 0.94 & $3-6.5$ & $-1.35(-4.49-1.80)$ & 0.4 & $3-6.5$ & $-0.66(-3.08-1.76)$ & 0.59 \\
\hline & $7-10.5$ & $1.58(-1.81-4.98)$ & 0.36 & $7-10.5$ & $-1.45(-4.97-2.07)$ & 0.42 & $7-10.5$ & $-1.35(-4.02-1.33)$ & 0.32 \\
\hline & $11-19.5$ & $-0.60(-4.22-3.02)$ & 0.75 & $11-19.5$ & $0.07(-3.65-3.79)$ & 0.97 & $11-19.5$ & $-1.29(-4.14-1.56)$ & 0.38 \\
\hline & & Trend $\mathrm{P}=0.92$ & & & Trend $\mathrm{P}=0.85$ & & & Trend $\mathrm{P}=0.39$ & \\
\hline
\end{tabular}

(b)

\begin{tabular}{|c|c|c|c|c|c|c|c|c|c|}
\hline & \multicolumn{3}{|c|}{ State anxiety } & \multicolumn{3}{|c|}{ Trait anxiety } & \multicolumn{3}{|c|}{ SDS } \\
\hline & $\begin{array}{l}\text { Range of the } \\
\text { category }\end{array}$ & $\begin{array}{c}\text { Odds ratio ( } 95 \% \\
\text { confidence interval) }\end{array}$ & $p$ & $\begin{array}{l}\text { Range of the } \\
\text { category }\end{array}$ & $\begin{array}{c}\text { Odds ratio ( } 95 \% \\
\text { confidence interval) }\end{array}$ & $p$ & $\begin{array}{l}\text { Range of the } \\
\text { category }\end{array}$ & $\begin{array}{c}\text { Odds ratio }(95 \% \\
\text { confidence interval) }\end{array}$ & $p$ \\
\hline \multirow[t]{2}{*}{ Qi-deficiency } & $0-9$ & 1 (referent) & & $0-9$ & 1 (referent) & & $0-9$ & 1 (referent) & \\
\hline & $10-14$ & $4.83(0.27-9.39)$ & 0.04 & $10-14$ & $6.37(1.68-11.07)$ & $<0.01$ & $10-14$ & $6.17(2.73-9.61)$ & $<0.001$ \\
\hline
\end{tabular}




\begin{tabular}{|c|c|c|c|c|c|c|c|c|c|}
\hline & $15-19$ & $4.58(-0.13-9.29)$ & 0.06 & $15-19$ & $7.59(2.79-12.39)$ & $<0.01$ & $15-19$ & $8.47(4.88-12.06)$ & $<0.001$ \\
\hline & $20-30$ & $9.29(4.08-14.49)$ & $<0.001$ & $20-30$ & $10.04(4.68-15.40)$ & $<0.001$ & $20-30$ & $11.02(7.09-14.96)$ & $<0.001$ \\
\hline & & Trend $\mathrm{P}<0.01$ & & & Trend $\mathrm{P}<0.001$ & & & Trend $\mathrm{P}<0.001$ & \\
\hline & $0-8$ & 1 (referent) & & $0-8$ & 1 (referent) & & $0-8$ & 1 (referent) & \\
\hline & $9-17$ & $2.24(-2.20-6.69)$ & 0.32 & $9-17$ & $6.25(1.69-10.81)$ & $<0.01$ & $9-17$ & $5.09(1.75-8.43)$ & $<0.01$ \\
\hline Qi-stagnation & $18-26$ & $4.02(-0.68-8.72)$ & 0.09 & $18-26$ & $9.17(4.29-14.04)$ & $<0.001$ & $18-26$ & $5.27(1.66-8.89)$ & $<0.01$ \\
\hline & $27-40$ & $4.05(-1.40-9.50)$ & 0.15 & $27-40$ & $6.89(1.35-12.43)$ & 0.01 & $27-40$ & $6.26(2.18-10.35)$ & $<0.01$ \\
\hline & & Trend $\mathrm{P}=0.17$ & & & Trend $\mathrm{P}=0.03$ & & & Trend $\mathrm{P}=0.01$ & \\
\hline & $0-7$ & 1 (referent) & & $0-7$ & 1 (referent) & & $0-7$ & 1 (referent) & \\
\hline & $8-13$ & $1.02(-3.27-5.31)$ & 0.64 & $8-13$ & $0.96(-3.56-5.48)$ & 0.68 & $8-13$ & $2.27(-0.99-5.52)$ & 0.17 \\
\hline Qi-flowback & $14-19$ & $-0.41(-5.13-4.31)$ & 0.86 & $14-19$ & $-0.41(-5.28-4.46)$ & 0.87 & $14-19$ & $0.88(-2.62-4.39)$ & 0.62 \\
\hline & $20-36$ & $6.94(1.89-11.99)$ & $<0.01$ & $20-36$ & $3.21(-2.08-8.50)$ & 0.23 & $20-36$ & $4.82(1.00-8.65)$ & 0.01 \\
\hline & & Trend $\mathrm{P}=0.02$ & & & Trend $\mathrm{P}=0.41$ & & & Trend $\mathrm{P}=0.05$ & \\
\hline & $0-6$ & 1 (referent) & & $0-6$ & 1 (referent) & & $0-6$ & 1 (referent) & \\
\hline & $7-12$ & $5.47(1.30-9.65)$ & 0.01 & $7-12$ & $2.61(-1.61-6.83)$ & 0.23 & $7-12$ & $3.33(0.16-6.50)$ & 0.04 \\
\hline Blood deficiency & $13-16$ & $6.08(1.39-10.78)$ & 0.01 & $13-16$ & $3.07(-1.65-7.79)$ & 0.2 & $13-16$ & $3.29(-0.22-6.81)$ & 0.07 \\
\hline & $17-28$ & $6.76(1.74-11.77)$ & $<0.01$ & $17-28$ & $5.46(0.34-10.58)$ & 0.04 & $17-28$ & $4.38(0.58-8.18)$ & 0.02 \\
\hline & & Trend $\mathrm{P}=0.01$ & & & Trend $\mathrm{P}=0.06$ & & & Trend $\mathrm{P}=0.03$ & \\
\hline & $0-2.5$ & 1 (referent) & & $0-2.5$ & 1 (referent) & & $0-2.5$ & 1 (referent) & \\
\hline & $3-5$ & $-0.67(-4.56-3.21)$ & 0.73 & $3-5$ & $-0.88(-4.82-3.06)$ & 0.66 & $3-5$ & $-1.70(-4.69-1.30)$ & 0.27 \\
\hline Fluid retention & $5.5-7.5$ & $-3.87(-8.80-1.05)$ & 0.12 & $5.5-7.5$ & $0.04(-5.05-5.13)$ & 0.99 & $5.5-7.5$ & $-2.63(-6.39-1.13)$ & 0.17 \\
\hline & $8-20.5$ & $-3.24(-7.78-1.29)$ & 0.16 & $8-20.5$ & $0.05(-4.60-4.70)$ & 0.98 & $8-20.5$ & $-1.20(-4.63-2.23)$ & 0.49 \\
\hline & & Trend $\mathrm{P}=0.34$ & & & Trend $\mathrm{P}=0.86$ & & & Trend $\mathrm{P}=0.82$ & \\
\hline
\end{tabular}



(a)

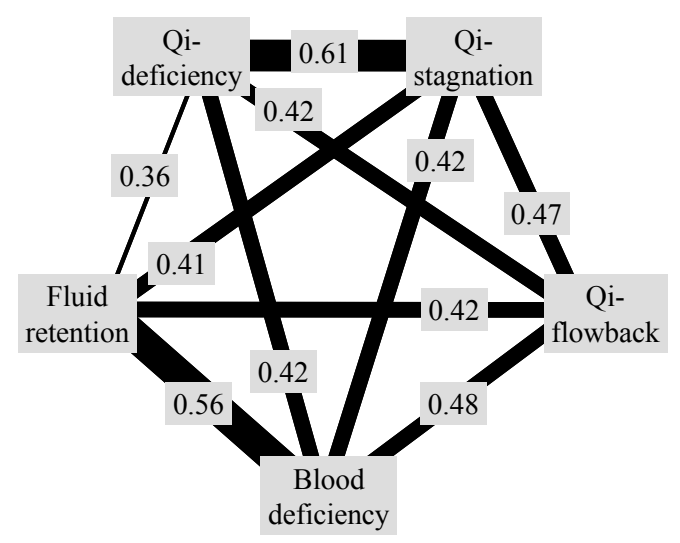

(b)

Figure 1.

Associations among Qi-blood-fluid patterns in females ( $a$ ) and males (b). Thickness of lines shows correlation coefficient. $p$ was less than 0.0001 between every pair. 
and Qi-stagnation in males and between blood deficiency and fluid retention in both sexes. Qi-patterns, especially Qi-deficiency and Qi-stagnation in both sexes and blood deficiency in males were associated with either major depression or high SDS scores. Qi-deficiency in both sexes, Qi-flowback in females, and blood deficiency in males, were associated with anxiety disorders or high STAI scores. Qi-stagnation in females was associated with STAI-I and inversely associated with total anxiety disorders. Fluid retention was associated with eating disorder in females. Qi-flowback and blood deficiency in females were associated with somatoform disorders. Qi-flowback in females was associated with irritable bowel syndrome.

Depression has been considered attributable to Qi-stagnation (Qiao et al., 2007; Wang et al., 2009; Yu et al., 2007; Zhou et al., 2006) and it is the case in the present study. However, our study indicated a stronger association between Qi-deficiency and major depression than Qi-stagnation in males. This result coincides with the stronger association between Qi-deficiency scores and SDS scores than between Qi-stagnation and SDS scores in males. Thus, resupply of Qi is important in treating male patients in the depressive state. The association with whole mood disorders was similar to that with major depression, since major depression made up $75 \%$ of the whole mood disorders.

There have been few reports on oriental conditions in patients with depression. Yang et al. gave acupuncture to patients with depression in view of their Qi-stagnation, blood stagnation, and Yan-deficiency, which is a combination of Qi-deficiency and cold constitution (Yang \& Zhi, 1994). Qi-stagnation is reportedly associated with depression in more than half of the cases (Zhou, Chen, \& Mei, 2006). In the present study, strong (more than 0.4 ) correlations with the SDS score, which reflect the extent of the psychological aspect of the depressive state, were observed in not only Qi-stagnation but also Qi-deficiency and Qi-flowback scores in both sexes. In treating the depressive state, re-supply of Qi or reducing Qi-flowback would also be effective as well as disobliteration of Qi-stagnation.

In the present study, examination of the association between depression and the chief complaints and KMI suggested that the strong association between depression and Qi-deficiency shown in Table 3 may be attributed to the strong association between depression and appetite loss, sensitivity to noise, hypobulia, and general fatigue among criteria for Qi-deficiency. The strong association between depression and Qi-stagnation could be attributed to the strong association between depression and hypobulia, appetite loss, headache/head discomfort, melancholy, unpleasant sensation of laryngopharynx, chest oppression, epigastric discomfort, a diversity of symptoms, and dependency of symptoms on mood.

The strong association between SDS scores and Qi-flowback is considered attributable to the strong association between depression and glow/chills, nausea/vomiting, headache, palpitation, sensitivity to noise, feeling of cold, diaphoresis, and abrupt perspiration, which are Qi-flowback symptoms. These symptoms also exist in the criteria for panic disorder, which often coexists with major depression. Recently, the coexistence and similarity between depression and panic disorder have been reported, which is an entity called "anxious depression in panic disorder." Although no association between panic disorder and Qi-flowback was detected in the present study, such comorbidity may account for the association between SDS scores and Qi-flowback. Since Qi-flowback was associated with not only SDS scores but also STAI scores, anxiety disorders and generalized anxiety disorder in females, depression, and panic disor- der might share such common pathological mechanisms as to be explained as Qi-flowback.

Qi-flowback in females was associated with high STAI scores, anxiety disorders, and generalized anxiety disorder. This result is considered because fear is included in the criteria of both Qi-flowback and generalized anxiety disorder.

A gender difference was observed in the association between anxiety and Qi-blood-fluid patterns. In females, all the Qi- patterns i.e. Qi-deficiency, Qi-stagnation, and Qi-flowback were associated with high State anxiety scores, high Trait anxiety scores, whole anxiety disorder, or generalized anxiety disorder. In contrast, blood deficiency and fluid retention were not. In males, Qi-stagnation and Qi-flowback were not associated with anxiety. Instead, blood deficiency was associated with high State anxiety scores and generalized anxiety disorder. Especially, only blood deficiency was associated with generalized anxiety disorder in males. Since anxiety is not included in Terasawa's criteria for blood deficiency, blood deficiency would be difficult to be taken into account as a differential diagnosis. In treating male patients with anxiety, resupply of not only Qi but also blood would be important.

The extremely high association between eating disorders and fluid retention in females may be attributed to vomiting and edema resulting from hypoalimentation.

Strong correlations of more than 0.4 were observed only between Qi-deficiency and Qi-stagnation and among Qi-flowback, blood deficiency, and fluid retention in females. The strong correlation between Qi-deficiency and Qi-stagnation can be explained by the fact that both are elements of depression as mentioned above. Besides, since some KMI questions and chief complaints were assigned to plural kinds of Qi-blood-fluid patterns, the least common denominators were attributed to some strong correlations between Qi-blood-fluid patterns. For example, hypobulia and anorexia as chief complaints or in KMI were common between Qi-deficiency and Qi-stagnation. This commonality is supposed to account for the strong correlation between Qi-deficiency and Qi-stagnation.

Higher correlations were also observed between Qi-deficiency and Qi-flowback in males, although there was only one common term. The comorbidity of Qi-deficiency and Qi-flowback in the present study is directly in line with the previous report that rectification of Qi without supply of Qi, that is treatment for Qi-flowback without treatment for Qi-defi- ciency, may exacerbate gastrointestinal motor dysfunction due to Qi-flowback (Mori, 1998).

Strong correlation coefficients were observed among Qiflowback, blood deficiency, and fluid retention, although Qi-flowback and blood deficiency have no common terms among their diagnostic criteria. The comorbidity of these three can be explained as elements of panic disorder. Many symptoms included in the criteria of panic disorder, i.e. numbness, diaphoresis, palpitation, dizziness, nausea, and chills belong to Qi-flowback, blood deficiency, or fluid retention. The pathology common to the basis of panic disorder may account for the strong correlations among Qi-flowback, blood deficiency, and fluid retention. When treating patients with atypical panic symptoms, diagnosis along the oriental longitudinal axes in addition to the occidental horizontal axes may be of assistance.

Among Qi-blood-fluid patterns included in Terasawa's Qi-blood-fluid scoring system, only blood-stagnation scores could not be calculated, since most of blood-stagnation score consists of physical examinations such as tenderness around the umbilicus. This is the limitation of the present study. A further investigation with physical examination is necessary, since 
blood-stagnation has been reported to be associated with various kinds of chronic disorders, especially those with fixed symptoms. Chronic blood stagnation leads to blood deficiency. In the present study, blood-defi- ciency was shown to be highly associated with somatoform disorders, most of which were pain disorder. This result coincides with the previous reports that that blood pattern including blood stagnation is associated with chronic pain, especially chronic pelvic pain in females (Chilla, Knusel, Zollikofer Ch, Huber, \& Kubik-Huch, 2006; Ganeshan et al., 2007).

The specific associations between the Qi-blood-fluid patterns and some occidental diseases or psychological tests, and the specific correlations among the Qi-blood-fluid patterns indicated in the present study may be advantageous for alternative medical treatment in an oriental manner for depression, anxiety disorder, eating disorder, somatoform disorder and irritable bowel syndrome.

\section{References}

Chan, J., Briscomb, D., Waterhouse, E., \& Cannaby, A. M. (2002). An uncontrolled pilot study of HT7 for 'stress'. Acupuncture in Medicine, 20, 74-77. doi:10.1136/aim.20.2-3.74

Chilla, B. K., Knusel, P. R., Zollikofer, C. L., Huber, T., \& Kubik-Huch, R. A. (2006). [Pelvic congestion syndrom]. Praxis, 95, 1583-1588. doi:10.1024/1661-8157.95.41.1583

Ganeshan, A., Upponi, S., Hon, L. Q., Uthappa, M. C., Warakaulle, D. R., \& Uberoi, R. (2007). Chronic pelvic pain due to pelvic congestion syndrome: the role of diagnostic and interventional radiology. Cardiovascular and Interventional Radiology, 30, 1105-1111. doi:10.1007/s00270-007-9160-0

Matsuoka, Y. (1990). KMI. In T. Kawano, H. Suematsu and R. Arazato (Eds.), Psychological Test for Psychosomatic Medicine (pp. 45-49). Tokyo: Asakura Publications.

Mori, Y. (1998). Composition and application of kampo formulas (2nd ed.): Ishiyaku Publications.

Nakasato, K. (1990). STAI. In T. Kawano, H. Suematsu and R. Arazato (Eds.), Psychological Test for Psychosomatic Medicine (pp. 27-31). Tokyo: Asakura Publications.

Spielberger, C. D., Gorsuch, R. L., \& Lushene, R. E. (1970). STAI manual. Palo Alto: Consulting Psychologist Press.

Terasawa, K. (1990). Japanese Kampo medicine with cases. Tokyo: Igaku Syoin Publications.

Terasawa, K. (2004). Evidence-based reconstruction of Kampo medicine: Part II-the concept of sho. Evidence-Based Complementary and Alternative Medicine, 1, 119-123. doi:10.1093/ecam/neh022

Wang, X. J., Wang, L. L., Qiao, H. F., \& Li, J. B. (2009). Observation on effective characteristics of acupuncture combined with medicine on depression of different syndrome types. Chinese Acupuncture \& Moxibustion, 29, 699-702.

Yang, Y., \& Zhi, D. (1994). Effects of acupuncture hypothermia and its relationship to changes of AVP contents in the plasma and CSF in the rabbits. Chen Tzu Yen Chiu Acupuncture Research, 19, 56-59.

Yuasa, Y. (1999). The nature and functions of Qi-energy in psychosomatic system. Japanese Journal of Psychosomatic Medicine, 39, 1926.

Zhou, L., Chen, W. K., \& Mei, X. Y. (2006). Study on the characteristics of population distribution of TCM syndromes and its related factors in patients of depression. Chinese Journal of Integrated Traditional and Western Medicine, 26, 106-109.

Zung, W. W., Richards, C. B., \& Short, M. J. (1965). Self-rating depression scale in an outpatient clinic. Further validation of the SDS Archives of General Psychiatry, 13, 508-515. 
Appendix 1.

Terasawa's Qi-blood-fluid scoring system.

\begin{tabular}{|c|c|c|c|c|c|c|c|c|c|}
\hline \multicolumn{2}{|c|}{ Qi-deficiency } & \multicolumn{2}{|l|}{ Qi-stagnation } & \multicolumn{2}{|l|}{ Qi-flowback } & \multicolumn{2}{|c|}{ blood-deficiency } & \multicolumn{2}{|l|}{ fluid-retention } \\
\hline symptom & score & symptom & score & symptom & score & symptom & score & symptom & score \\
\hline general fatigue & 10 & depressive mood & 18 & $\begin{array}{l}\text { cold constitution } \\
\text { and hot flush }\end{array}$ & 14 & $\begin{array}{l}\text { retardation of } \\
\text { thought }\end{array}$ & 6 & heaviness of body & 3 \\
\hline hypobulia & 10 & heavy-headed feeling & 8 & palpitation attack & 8 & $\begin{array}{l}\text { early-morning } \\
\text { awakening }\end{array}$ & 6 & systaltic headache & 4 \\
\hline easy fatigability & 10 & $\begin{array}{l}\text { unpleasant sensation } \\
\text { of laryngopharynx }\end{array}$ & 12 & sporadic headache & 8 & asthenopia & 12 & heavy-headed feeling & 3 \\
\hline hypersomnia & 6 & chest oppression & 8 & vomiting & 8 & dizziness & 8 & carsickness & 5 \\
\hline anorexia & 4 & $\begin{array}{l}\text { hypochondrial } \\
\text { oppression }\end{array}$ & 8 & cough & 10 & cramp & 10 & dizziness & 5 \\
\hline $\begin{array}{l}\text { vulnerability to } \\
\text { cold }\end{array}$ & 8 & abdominal fullness & 8 & $\begin{array}{l}\text { abrupt abdominal } \\
\text { pain }\end{array}$ & 6 & slight menorrhea & 6 & $\begin{array}{l}\text { dizzy feeling on } \\
\text { standing up }\end{array}$ & 5 \\
\hline scariness & 4 & $\begin{array}{l}\text { counterchanging of } \\
\text { symptoms }\end{array}$ & 8 & scariness & 6 & pale complexion & 10 & watery rhinorrhea & 3 \\
\hline hollow voice & 6 & difficulty in uprising & 8 & impatience & 8 & alopecia & 8 & ptyalism & 3 \\
\hline pale tongue & 8 & abdominal wind & 6 & flush & 10 & xeroderma & 14 & foamy sputum & 4 \\
\hline vacuous pulse & 8 & burping & 4 & $\begin{array}{l}\text { periomphal } \\
\text { pulsation }\end{array}$ & 14 & ungual incisure & 8 & nausea & 3 \\
\hline flaccidity & 8 & sense of residual urine & 4 & crural chills & 4 & paresthesia & 6 & rugitus & 3 \\
\hline uterine prolapse & 10 & tympanicity & 8 & $\begin{array}{l}\text { palmar and plantar } \\
\text { diaphoresis }\end{array}$ & 4 & $\begin{array}{l}\text { rectus abdominis } \\
\text { spasm }\end{array}$ & 6 & arthral tightening & 7 \\
\hline $\begin{array}{l}\text { hypogastric } \\
\text { flaccidity }\end{array}$ & 6 & & & & & & & edema & 15 \\
\hline \multirow[t]{5}{*}{ diarrhea } & 4 & & & & & & & effusion & 15 \\
\hline & & & & & & & & periomphal pulsation & 5 \\
\hline & & & & & & & & watery diarrhea & 5 \\
\hline & & & & & & & & oliguria & 7 \\
\hline & & & & & & & & polyuria & 5 \\
\hline
\end{tabular}

Appendix 2.

Correspondence between symptoms described as chief complaints and Qi-blood-fluid patterns in terasawa's scoring system.

\begin{tabular}{|c|c|c|c|c|c|}
\hline Chief complaint & Qi-deficiency & Qi-stagnation & Qi-flowback & Blood-deficiency & Fluid-retention \\
\hline Abdominal distention & & $\circ$ & & & \\
\hline Abdominal pain & & & $\circ$ & & \\
\hline Abdominal wind & & $\circ$ & & & \\
\hline Alopecia & & & & $\circ$ & \\
\hline Anorexia & $\circ$ & $\circ$ & & & \\
\hline Appendicular dindle & & & & ० & \\
\hline Arthral tightening & & & & & $\circ$ \\
\hline Blurred vision & & & & $\circ$ & \\
\hline Burping & & $\circ$ & & & \\
\hline Chest discomfort & & $\circ$ & & & \\
\hline Chest oppression & & $\circ$ & & & \\
\hline Cold constitution and hot flush & & & o & & \\
\hline
\end{tabular}


Convulsion

Cough

Cramp

Diaphoresis

Diarrhea

Difficulty in falling asleep

Difficulty in uprising

Disruption of sleep

Dizziness

Dizzy feeling on standing up

Dysgeusia

Early-morning awakening

\section{Edema}

Epigastric discomfort

Epigastric oppression

Excessive dream

Feeling of heaviness

Feeling of laryngeal occlusion

Flush

Frequent urination

General fatigue

Headache

Heavy-headed feeling

Hollow voice

Hypersomnia

Hypobulia

Hypoesthesia

Impatience

Irregular menstruation

Manual chill

Melancholy

Nausea

Nightmare

Palmar and plantar diaphoresis

\section{Palpitation}

Retardation of thought

Slight menorrhea

Somnolence in the daytime

Systaltic headache 
Appendix 3.

Correspondence between questions in KMI, and Qi-blood-fluid patterns in terasawa's scoring system.

\begin{tabular}{|c|c|c|c|c|c|c|}
\hline KMI Question number & Symptom & Qi-deficiency & Qi-stagnation & Qi-flowback & Blood-deficiency & Fluid-retention \\
\hline B-02 & Impatience & & & $\circ$ & & \\
\hline B-04 & Nightmares & & & & $\circ$ & \\
\hline B-11 & Retardation of thought & & & & $\circ$ & \\
\hline B-12 & Hypobulia & $\circ$ & $\circ$ & & & \\
\hline B-21 & Irritability & & & $\circ$ & & \\
\hline B-31 & Counterchanging of symptoms & & $\circ$ & & & \\
\hline B-32 & Fluctuation of symptoms & & $\circ$ & & & \\
\hline B-42 & Melancholy & & $\circ$ & & & \\
\hline B-43 & Hypobulia & $\circ$ & $\circ$ & & & \\
\hline $\mathrm{C}-03$ & General fatigue & $\circ$ & & & & \\
\hline C-05 & Easy fatigability & & & & & \\
\hline $\mathrm{C}-07$ & Anorexia & $\circ$ & $\circ$ & & & \\
\hline C-09 & Palpitation & & & $\circ$ & & \\
\hline $\mathrm{C}-11$ & Chest oppression & & $\circ$ & & & \\
\hline $\mathrm{C}-12$ & Palpitation & & & $\circ$ & & \\
\hline $\mathrm{C}-14$ & Cold constitution and hot flush & & & $\circ$ & & \\
\hline $\mathrm{C}-15$ & Diaphoresis & & & $\circ$ & & \\
\hline $\mathrm{C}-16$ & Edema & & & & & $\circ$ \\
\hline $\mathrm{C}-17$ & Crural chill & & & $\circ$ & & \\
\hline $\mathrm{C}-18$ & Asthenopia & & & & $\circ$ & \\
\hline $\mathrm{C}-21$ & Feeling of laryngeal occlusion & & $\circ$ & & & \\
\hline $\mathrm{C}-22$ & Epigastric oppression & & & & & \\
\hline $\mathrm{C}-23$ & Nausea & & & $\circ$ & & $\circ$ \\
\hline $\mathrm{C}-24$ & Rugitus & & & & & $\circ$ \\
\hline $\mathrm{C}-26$ & Abrupt abdominal pain & & & $\circ$ & & \\
\hline $\mathrm{C}-31$ & Frequent urination & & & & & $\circ$ \\
\hline $\mathrm{C}-35$ & Irregular menstruation & & & & $\circ$ & \\
\hline $\mathrm{C}-36$ & Retardation of thought & & & & $\circ$ & \\
\hline $\mathrm{C}-37$ & Heavy-headed feeling & & $\circ$ & $\circ$ & & $\circ$ \\
\hline C-38 & Dizziness & & & & $\circ$ & $\circ$ \\
\hline C-42 & Dindle or hypoesthesia & & & & $\circ$ & \\
\hline C- 43 & Fasciculation & & & & $\circ$ & \\
\hline C-44 & Difficulty in falling asleep & & & & $\circ$ & \\
\hline $\mathrm{C}-45$ & Disruption of sleep & & & & $\circ$ & \\
\hline C- -46 & Excessive dreaming & & & & $\circ$ & \\
\hline C-47 & Nightmares & & & & $\circ$ & \\
\hline
\end{tabular}

